\title{
Cardiac Fibroblasts and Cellular Cross Talk in Heart Failure
}

\author{
Burns C. Blaxall • Troy A. Baudino • \\ Lorrie A. Kirshenbaum
}

Received: 20 September 2012 / Accepted: 10 October 2012 /Published online: 8 November 2012

(C) Springer Science+Business Media New York 2012

Cardiovascular disease represents the leading cause of death in the USA and throughout much of the world. Heart failure is a common end-stage manifestation of most cardiovascular complications and is a devastating disease with poor prognosis. Unfortunately, there is a rapidly growing societal burden of heart failure commensurate with widespread obesity, diabetes, and an aging population both here and abroad. As such, increased understanding of the underlying mechanisms of heart failure, including those that may lead to development of improved cardiovascular therapeutics, is desperately needed.

Traditionally, investigation of differentiated mammalian tissues (e.g., the heart and brain) has focused on improving the "functional" cell of that tissue (e.g., cardiomyocyte and

\section{B. C. Blaxall $(\bowtie)$}

The Heart Institute, Molecular Cardiovascular Biology, Cincinnati Children's Hospital Medical Center,

3333 Burnet Ave, MLC 7020, Cincinnati, OH 45229, USA

e-mail: burns.blaxall@cchmc.org

T. A. Baudino

Cardiovascular Research Institute, Department of Medicine,

Division of Molecular Cardiology, Texas A\&M

University College of Medicine,

1901 South 1st Street, Building 205, Room 1R34,

Temple, TX 76504, USA

e-mail: tbaudino@medicine.tamhsc.edu

L. A. Kirshenbaum

Faculty of Medicine, Departments of Physiology and

Pharmacology \& Therapeutics, Institute of Cardiovascular

Sciences, St. Boniface General Hospital Research Centre,

University of Manitoba,

Rm 3016, 351 Tache Avenue,

Winnipeg, Manitoba, Canada R2H2A6

e-mail: LKirshenbaum@sbrc.ca neuron). Substantial research efforts have been focused on understanding the functional cell and how to possibly improve its function in a diseased state. However, there is a growing appreciation for the important role of "nonfunctional" cells in a variety of differentiated tissues (e.g., cardiac fibroblast and microglial cell). Over the past decade, a relatively new area of cardiovascular research has focused on elucidating the importance of non-myocyte cells in the pathogenesis of heart disease. The overarching purpose of this issue of the journal is to highlight, in a single collection of topical reviews, the emerging and important functional role of myocardial intercellular communication in the development and progression of heart failure. Although the approaches described in this issue by no means constitute a comprehensive review of all current cell types and modes of cardiac intercellular communication, we sought to highlight the breadth of novel approaches to understanding heart failure progression via cellular cross talk.

A variety of non-myocyte cells populate the myocardium, in which the cardiomyocyte represents a nearminority cell number population in the heart. Much of this issue focuses on a major non-cardiomyocyte cell in the heart, namely, the cardiac fibroblast. The importance of the fibroblast's developmental origin in determining both its physiologic role and electrophysiologic potential is reviewed. The emerging significance of the transition of the cardiac fibroblast to the pathologic, paracrine phenotype of a myofibroblast is reviewed, including the role of transcription factors and the renin-angiotensin system in this process. The dynamic role of the cardiac fibroblast in cardiac development and disease as well as the role of the extracellular matrix in facilitating the myofibroblast transition post-myocardial infarction are also summarized. 
Fibroblasts and a role for intercellular communication in the heart are also highlighted, including the dynamic cross talk between cardiomyocytes and fibroblasts via follistatin-like 3 , protease-activated receptor 1 , and others. Finally, overviews of the impact of cardiomyocyte-fibroblast interactions on the coronary vasculature as well as the important role of the innate immune system in coordinating cardiac cellular response to injury are included.
The challenges to enhancing our understanding of heart failure toward developing new treatments are indeed daunting, as evidenced by the paucity of new therapeutics. However, with this issue of the journal, we wish to highlight the emerging role of cardiac intercellular communication in cardiovascular disease, possibly opening new avenues for the development of novel treatments for this devastating disease. 